EVS28

KINTEX, Korea, May 3-6, 2015

\title{
The provision of public recharging infrastructure for Electric Vehicles in the UK - is there a business case?
}

\author{
Josey Wardle ${ }^{1,3}$, Prof Phil Blythe ${ }^{1}$, Dr Jane Gibbon ${ }^{2}$, Dr Colin Herron ${ }^{3}$, Dr Yvonne Hübner ${ }^{1}$
}

${ }^{1}$ Newcastle University, Transport Operations Research Group (TORG), Civil Engineering and Geosciences, Newcastle Upon Tyne, NE1 7RU, UK, email:

${ }^{2}$ Newcastle University Business School, 5 Barrack Road, Newcastle upon Tyne, NE1 4SE, UK

${ }^{3}$ Zero Carbon Futures, SASMI, Washington Road, Sunderland, SR5 3HE, UK

\begin{abstract}
The UK has been one of the most advanced countries in Europe for the demonstration of electric vehicles $(\mathrm{EV})$ and the introduction of the supporting recharging infrastructure. Much of the UK's EV recharging estate was created and is operated under public subsidy, in order to seed the marketplace for further EV and recharging equipment adoption. This paper addresses the fact that subsidies for the operation of this infrastructure are coming to an end, which is likely to affect EV drivers' recharging behaviour. As the public funding ceases the infrastructure owners must find other ways to cover the on-going costs of operation and recover capital investments made, in order to provide a continuing and viable service to EV drivers. However, actual uptake of EVs and therefore demand for recharging has been lower than the arguably over optimistic predictions made in 2010 when the subsidies began. The difficulty of covering operating costs with inaccurate EV forecast figures is compounded by factors including asset life and ownership costs, recharging locations, vehicle and charging specifications, vehicle usage patterns and regional demographics. The introduction of fees for recharging at a level which EV drivers are willing to pay is unlikely to enable infrastructure owners to recoup their full costs using conventional business models. A Social and Environmental accounting model could be developed to help inform decision making for the public recharging business case. This paper gives an overview of the findings from the UK supplemented by experience from Republic of Ireland, and comments on the impact of inaccurate EV sales predictions and early changes in recharging behaviour resulting from reduction in subsidies.
\end{abstract}

Keywords: electric vehicles, infrastructure, recharging, subsidies, business case 


\section{Introduction}

Transport is a major source of greenhouse gas emissions, which cause global climate change. Therefore many European countries have introduced measures aimed at reducing transport emissions. The European Union's (EU) Clean Power for Transport policy[1] seeks to break Europe's dependence on oil for Transport, and therefore sets out a package of measures to facilitate the development of a single market for alternative fuels for transport in Europe. The EU's proposal for a Directive on the Deployment of Alternative Fuels Infrastructure[2] was released in January 2013 requiring Member States to adopt national policy frameworks for the market development of alternative fuels and their infrastructure.

The UK government is encouraging Ultra Low Emission Vehicle (ULEV) adoption, which includes both pure EV and plug-in hybrid vehicles (PHEV), through a number of incentives operated by its Office for Low Emission Vehicles (OLEV). This includes incentives for plug-in cars[3], vans[4], and public fleets. The UK government also considers the availability of public charge points as necessary to encourage and enable the uptake of plug-in ULEVs, which led to the creation of its Plugged In Places (PIP)[5] infrastructure subsidy programme in 2010. The PIP programme installed 6,483 charge points in 8 regions of the UK with the specific objectives of investigating different recharging strategies, evaluating recharging technologies, investigating user behaviour and advancing common standards. The results from the PIP programme were used to inform the UK government's ULEV strategy entitled "Driving the Future Today"[6] released in Sept 2013. This includes the fundamental requirements for providing plug-in vehicle recharging infrastructure and the importance of public recharging facilities to $\mathrm{EV}$ buyers.

The Republic of Ireland has provided similar incentives for EVs and infrastructure operated by ESB's[7] ecars[8] programme.

Various recharging infrastructure projects across Europe are currently benefitting from EU funding, including the Rapid Charge Network[9] $(\mathrm{RCN})$. All of these projects are supporting the EU towards its goal for the de-carbonization of transport and contributing to the target of a $60 \%$ reduction of $\mathrm{CO}^{2}$ emissions from transport by 2050.

This is a classic "Chicken and Egg" conundrum. Recharging infrastructure is required to enable drivers to consider buying EVs. Consumers require the comfort of knowing they can recharge if and when required, even if they subsequently don't often use the public facilities provided to meet those perceived needs[10]. Recharging infrastructure falls outside of the EV manufacturers' traditional area of activity, creating an ongoing debate about who is responsible for recharging provision and ownership.

The purpose of this paper is to illustrate the difficulties in finding a business case for the ongoing provision of public recharging infrastructure, created and operated to date under subsidies which are now falling away, without sufficient demand to cover operating costs. The paper will present and discuss the current ongoing research to consider what alternative business models could be brought to the sector to deliver a long term sustainable business model for the future roll-out of critical EV recharging infrastructure.

\section{$2 \quad$ Methodology}

A continuing longitudinal review of the impact of the North East England PIP (NE PIP) since 2010 has been used as the basis for this paper. The discussion has been further informed by results from Ireland's ESB ecars public infrastructure programme and early findings from the Rapid Charge Network (RCN) project's national study of the use of rapid charging infrastructure located along major highways of the UK and Ireland. This data has been used to inform both the supply and demand elements of the business case discussion.

Supply of recharging services is driven by the composition of recharging networks. Composition of early recharging networks delivered from 2010 by NE PIP and ESB ecars was driven by two things; the recharging technologies of the time and the availability of suitable locations at which to install charge points.

Recharging technology is driven by the EV manufacturers. In 2010 the majority of EVs in production required only $3 \mathrm{~kW}$ AC or the $50 \mathrm{~kW}$ DC Chademo rapid charging protocol. 7 and 22 
$\mathrm{kW}$ AC requirements followed, and in 2013 the Combo rapid charging protocol was introduced to the UK and Ireland with BMW's entry to the EV market. These new technologies presented problems for the early recharging networks, adding cost in the provision of new hardware and software and raising the issue of lost revenue opportunities as a result of different connector designs promoted by different EV manufacturers.

Suitable locations are driven by power availability, location, access, ownership, parking capacity, and visibility, each of which can be controlled by different stakeholders.

\subsection{ULEV adoption}

Demand for recharging services is driven by ULEV sales. The initial forecasts of likely ULEV sales shaped early recharging infrastructure provision in the UK and Ireland. Accurate forecasts are a critical factor in the infrastructure debate. These forecasts influence and can ensure the success or failure of business models for ongoing operation and any additional provision. Since the demand for recharging services is driven by the uptake of plug-in ULEV, the actual ULEV sales figures in the UK and Ireland have been monitored since 2010, in order to understand market growth and potential demand for recharging. The actual ULEV sales figures have fallen short of the predictions made in 2010 and therefore the demand for recharging services is also lower than predicted.

\subsection{The NE PIP project}

North East (NE) PIP project (NE PIP) created an integrated recharging network for EVs spanning a region of $8,600 \mathrm{~km}^{2}$ and a population of $2.6 \mathrm{~m}$ between April 2010 and June 2013. This recharging infrastructure has enabled EV journeys to become feasible across the region and into neighbouring regions as well as Europe. The project installed 1,163 charge points in public places, workplaces and in the homes of EV drivers across the region. The NE PIP public access and workplace charge points were operated by a subsidised recharging network, the funding for which ended in June 2013.

Detailed information from NE PIP was used to understand the existing charge point estate in NE England, its distribution and ownership, its capability and capacity, and the way it has been used to date. Usage data will continue to be analysed to understand ongoing recharging behaviour until 2016, to capture changes in behaviour after subsidies have ended and hosts are able to charge a fee for the recharging service. This information will also be used to model likely future demand.

The recharging estate includes a combination of 3, 7 and $22 \mathrm{~kW}$ AC charge points, and the first regional network of $50 \mathrm{~kW}$ DC rapid charge points in the UK, which enable EVs to be recharged to $80 \%$ of full state of charge in circa 30 minutes. 12 rapid chargers were installed close to main highways across the region to enable longer EV journeys and to provide added confidence to EV drivers.

Potential hosts were attracted to have charge points installed on their property by various levels of grant incentives covering equipment and installation costs. Charge points have consequently been installed in locations in accordance with demand from interested hosts. In exchange for this grant funding, each host provided free electricity and free parking to EV drivers during the three year trial period, which ended in June 2013. The charge point hosts now own the NE's EV recharging infrastructure which forms the NE recharging estate.

All publically accessible charge points were operated by a single network operator, Charge Your Car (CYC)[11]. CYC was funded to provide access to the entire NE recharging estate, as well as to provide customer service and charge point information via a live availability map on a dedicated website. EV drivers joined the NE PIP's CYC membership scheme at a cost of $£ 100$ $(€ 135 / \$ 154)$ per year or $£ 10(€ 13.50 / \$ 15.40)$ per month, in order to receive free electricity and parking whilst recharging, access to the website to plan their journeys and their own recharging records. In addition to this public and workplace infrastructure, the project also installed over 400 domestic chargers with captive cables for EV drivers in the region to use in their home environment.

\subsection{ESB ecars programme}

The Sustainable Energy Authority of Ireland (SEAI) operates a grant scheme providing an incentive of up to $€ 5,000$ to an $\mathrm{EV}$ purchaser. 
Alongside these vehicle incentives, ESB committed to provide the supporting recharging infrastructure and associated ICT support systems under a tripartite agreement with NissanRenault and the Irish Government, signed in February 2010. This budget has since been supplemented by funding from the Irish Energy Regulator (CER) and various EU projects, which enabled all costs to be covered until the end of 2014.

ESB ecars is providing free domestic chargers to the first 2,000 EV purchasers who qualify for the SEAI grant scheme. It has also deployed public rapid chargers in approximately 450 locations across the country, which are operated on the ecars charge point management system (CPMS). The aim is that all towns in the Republic of Ireland with a population of 1,500 or more will have at least one charging post serving the town. Data collected from the ecars CPMS is now being used to inform future planning.

In the knowledge that early funds were coming to an end, in September 2014 ESB ecars began to research business models to give return on the assets deployed. Currently the energy delivered in recharging transactions is provided free of charge to EV drivers, a deliberate incentive intended to enhance the attractiveness of EVs and increase uptake. The energy delivered is treated as transmission network losses. Irish energy suppliers are aware of this and, given its low value to date, will tolerate this for a short period of time. ESB own the charge point estate. Therefore ESB is funding the costs of operation going forward, including network operation and maintenance costs.

However, as EV sales increase and demand for recharging grows this arrangement will become unacceptable so an alternative business model is required.

\subsection{The Rapid Charge Network project (RCN)}

Early data from the RCN project is also being used to investigate the characteristics of rapid charging behaviour in more depth, in order to understand its role in the business model for public charge point provision.

RCN is an EU part-funded TEN-T project assessing the creation, use and business feasibility of a multi-standard, interoperable network of EV rapid charge points linking the UK with Republic of Ireland.

The RCN project is being delivered by a consortium of four major EV manufacturers Nissan, BMW, Renault and VW, complemented by the latest knowledge from Ireland's ESB ecars. These partners provide the balance of funding for the creation and operation of the infrastructure until December 2015.

RCN's strategic purpose is to accelerate the introduction of EV Rapid Charging infrastructure by studying adoption and use along over $1,100 \mathrm{~km}$ of TEN-T defined priority road routes across the UK and into Republic of Ireland. The RCN Project aims to install up to 74 multi-standard rapid chargers, each equipped with 3 charging outlets accommodating all 3 rapid charging protocols and connectors currently required by the volume European EV manufacturers, and then to study their operation and use until the end of 2015. The multi-standard rapid chargers incorporate the 44 $\mathrm{kW}$ DC Chademo and CCS, and $43 \mathrm{~kW}$ AC rapid charging protocols into one charging unit, which can recharge all standardised EVs to $80 \%$ of full battery capacity in circa 30 minutes. The chargers are located at motorway service stations and major retail facilities along main highways, and will link through major air and seaports to multi-modal transport solutions. The rapid chargers are operated free of charge to EV drivers until the end of 2015, under Ecotricity's Electric Highway network in the UK and ESB's ecars network in Ireland.

\section{Results}

\subsection{ULEV adoption}

The EU has set average new car fleet emission targets of $130 \mathrm{gCO}_{2} / \mathrm{km}$ for 2015 and $95 \mathrm{gCO}_{2} / \mathrm{km}$ by 2020[12]. Vehicle manufacturers will require significant adoption of ULEV in order to reach these targets and avoid financial penalties.

ACEA, the European Automobile Manufacturer's Association, has reported that 75,331 new ULEVs were registered in the EU during 2014, representing a $36.6 \%$ rise over 2013. Looking at the EU's major markets, the UK saw the largest increase over the year $(+300.8 \%)$, followed by Germany (+70.2\%) and France (+29.8\%) [13]. 


\subsubsection{UK}

The UK government has stated that its objective is to see a UK car fleet with effectively zero emissions by 2050 .

The UK government reports that 23,138 Plug-in Vehicle grants had been claimed by the end of 2014. In total 2014 claims were four times higher than 2013, and 1 in every 78 cars bought in the UK in December 2014 was a ULEV. This suggests that the UK market is in a high growth phase. This has been driven both by government incentives and the growing mix of attractive ULEV models available for sale in the UK.

923[14] of these ULEV were registered in the NE region by October 2014. However, NE PIP data tells us that additional ULEVs are in use through employee lease schemes in the region but these were registered in other regions under lease operators' addresses. For example, an additional 312 LEAF were in use by Nissan employees in NE England in Oct 2014. The combined total makes the region the third highest user of ULEV per head of population in the UK. This is an important finding since the region also has the lowest GVA per head of population in the UK and the lowest spend on transport.

\subsubsection{Republic of Ireland}

The Irish government targeted that $10 \%$ of the overall national fleet (circa 200k) cars should be electrified by 2020 . $643 \mathrm{EV}$ sales have been recorded up to January 2015, suggesting that this challenging target may not be reached due to the effects of the recession, unless sales can be significantly accelerated further.

However, EV sales in Republic of Ireland have increased over the period since the ecar programmes were launched. The same number of EVs were sold in January 2015 than were sold in the entirety of 2013.

Following a number of surveys both of the general population and EV drivers, ESB ecars has identified a number of reasons why EV ownership has increased in Republic of Ireland. EV related reasons include the greater variety of EV models available, and the total cost of EV ownership becoming more competitive with a reduction in EV prices. Infrastructure related reasons include the availability of a nation-wide rapid charging network as well as inter-urban rapid charging facilities. In particular a $15 \%$ increase in cross-border EV traffic has been recorded since the deployment of a TEN-T funded rapid charger network between the Republic of Ireland and its neighbour Northern Ireland, which is part of the UK.

\subsection{NE PIP}

Analysis of NE PIP usage data suggests that both the location and equipment type of charge points appears to drive recharging behaviour, which makes both important factors in the business model. NE PIP data has been reviewed to understand the composition of charge points within the estate, both by location and equipment type, as shown in Table 1 below.

Table 1: Composition of NE PIP estate by equipment and location types.

\begin{tabular}{|l|r|r|r|r|r|}
\hline \multirow{2}{*}{$\begin{array}{r}\text { Charging Equipment } \\
\text { types }\end{array}$} & \multicolumn{2}{|l|}{ Standard Chargers } & \multicolumn{2}{|c|}{ Rapid Chargers } & \\
\cline { 2 - 6 } & $\mathbf{3 ~ k W ~ A C}$ & $\mathbf{7 ~ k W ~ A C}$ & $\mathbf{2 2}$ kW AC & $\mathbf{5 0 ~ k W ~ D C}$ & \\
\hline Location types & & & & & \\
\hline Workplace & 60 & 261 & 0 & 0 & \\
\hline Public & 90 & 326 & 12 & 13 & \\
\hline Home chargers & 401 & 0 & 0 & 0 & \\
\hline Totals & $\mathbf{5 5 1}$ & $\mathbf{5 8 7}$ & $\mathbf{1 2}$ & $\mathbf{1 3}$ & $\mathbf{1 1 6 3}$ \\
\hline
\end{tabular}

Standard equipment delivers 3 or $7 \mathrm{~kW}$ of $\mathrm{AC}$ power. Rapid chargers deliver $22 \mathrm{~kW}$ AC or 50 $\mathrm{kW}$ DC power, recharging an $\mathrm{EV}$ in a much shorter time, which suggests that they may be more attractive to EV drivers and therefore a more valuable component of the estate than standard chargers. Standard chargers are much cheaper to buy and install but take much longer to charge an EV. Standard chargers make up the majority of the NE public infrastructure estate with rapid chargers providing only a small part of the public provision.

Usage data shows that the combined estate of 737 public and workplace chargers has delivered 81,594 recharging transactions and over $589 \mathrm{MWh}$ of energy to EV drivers up to the end 2014. This provides insight into the historic recharging demand from EV drivers in the region. Analysis suggests that rapid chargers are used more frequently and deliver more energy per recharging transaction than standard chargers, suggesting they are a more valuable component of the estate. The proportion of transactions broken down by location 
category is shown in Figure 1, and the energy delivered is displayed in Figure 2.

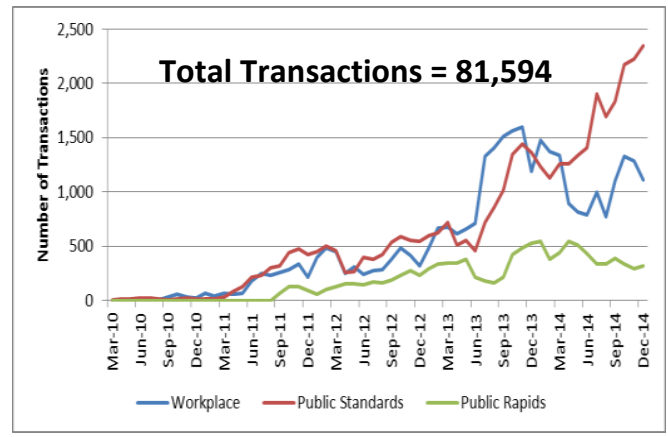

Figure 1: Transactions delivered by NE PIP charge point estate to Dec 2014.

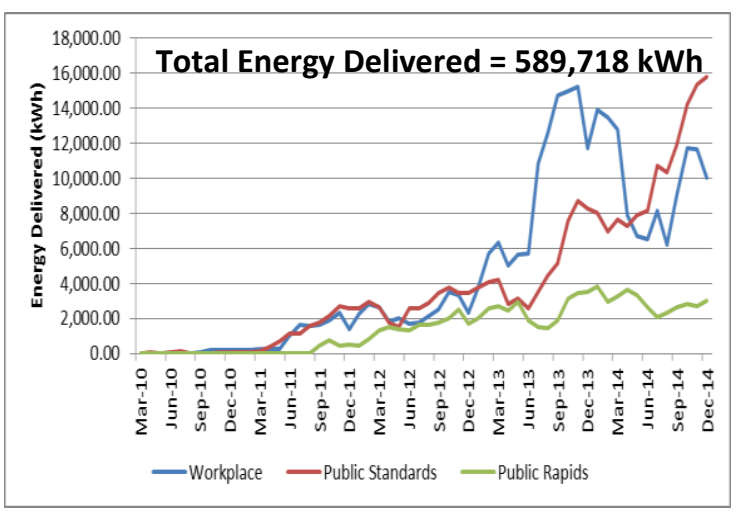

Figure 2: Energy delivered by NE PIP charge point estate to Dec 2014.

The rapid chargers have delivered a much higher proportion of the transactions $(15 \%)$ and total energy (16\%) than their composition proportion suggests (2\%). Conversely, the public standard chargers delivered a lower proportion of the total transactions (43\%) and energy (35\%) compared to their composition within the public and workplace estate $(55 \%)$. This suggests that $\mathrm{EV}$ owners would prefer to use rapid chargers if they are available and that there may be a risk to hosts and operators investing in standard charging infrastructure, as it may not be used and therefore the desired return on investment may not be achievable.

Usage patterns also differ throughout the week. Initial analysis indicates that recharging events on standard public chargers fell by $40 \%$ at the weekend. However recharging events on public rapid chargers remained relatively constant throughout the week, only falling by $17 \%$ at the weekend. Secondly, the energy transferred per charge event differs between the different charge point location types, as shown in Table 2. Public standard chargers deliver a lower average energy per transaction than workplace based equivalents, and rapid chargers deliver a higher average energy per transaction than standard chargers.

Since the number of recharging transactions and the energy delivered by them indicate the demand for charge point use, forecasts for future public charge point use have been reached by fitting linear trends to the historical data. Tables 2 and 3 illustrate that these forecasts show little likely increase in demand for public standard charging which make up the majority of the NE estate. However, forecasted demand for rapid charge point use shows a marked increase in quantity and average energy per transaction by Dec 2016.

Table 2: NE PIP changes in energy delivered per transaction and linear forecasts to Dec 2016

\begin{tabular}{|l|r|r|r|}
\hline \multirow{2}{*}{$\begin{array}{l}\text { Changes in chargepoint use \& } \\
\text { linear forecasts for future use }\end{array}$} & \multicolumn{3}{|c|}{ Average Energy delivered per Transaction } \\
\cline { 2 - 4 } & (kWh) \\
\cline { 2 - 5 } & $\begin{array}{c}\text { Dec 2012 } \\
\text { Actual }\end{array}$ & $\begin{array}{c}\text { Dec 2014 } \\
\text { Actual }\end{array}$ & $\begin{array}{r}\text { Dec 2016 } \\
\text { Forecast }\end{array}$ \\
\hline Public Standards & 6.38 & 6.75 & 8.00 \\
\hline Public Rapids & 7.41 & 9.47 & 14.25 \\
\hline Workplace & 7.31 & 9.05 & 14.00 \\
\hline
\end{tabular}

Table 3: NE PIP changes in transactions delivered per charge point and linear forecasts to Dec 2016

\begin{tabular}{|c|c|c|c|}
\hline \multirow{2}{*}{$\begin{array}{l}\text { Changes in chargepoint use \& } \\
\text { linear forecasts for future use }\end{array}$} & \multicolumn{3}{|c|}{ Monthly Transactions per Chargepoint } \\
\hline & $\begin{array}{c}\text { Dec } 2012 \\
\text { Actual }\end{array}$ & $\begin{array}{l}\text { Dec } 2014 \\
\text { Actual }\end{array}$ & $\begin{array}{l}\text { Dec } 2016 \\
\text { Forecast }\end{array}$ \\
\hline Public Standards & 1.31 & 5.64 & 5.89 \\
\hline Public Rapids & 9.75 & 13.42 & 25.00 \\
\hline Workplace & 1.00 & 3.45 & 6.07 \\
\hline
\end{tabular}

NE PIP data shows that the 12 Local Authorities (LA) in the region together own $80 \%$ of the public chargers. These LAs have social and environmental aims but are facing severe financial constraints imposed by increasing government cuts, so some have already begun to impose fees for charge point use. However, initial data shows a reduction in use of those charge points since fees were introduced, suggesting that EV drivers are sensitive to any change in recharging cost and are likely to change their behaviour as a result. Monitoring these changes is a key element of this research. The likely mismatch between charge point owners preferred fees and EV drivers 
willingness to pay suggests that a method to value the social and environmental benefits of charge point provision may help to ensure continuing provision of public charge points.

\subsection{ESB ecars}

ESB ecars has deployed over 1,800 charge points across Republic of Ireland up to January 2015, as shown in Table 4. All of the publically accessible estate is made up of rapid chargers. This infrastructure estate includes recharging equipment for a mixture of different rapid charging protocols and their associated connectors, as shown in Table 4.

Table 4: Composition of Irish charge point estate at Jan 2015

\begin{tabular}{|c|c|c|c|c|c|}
\hline \multirow[b]{2}{*}{$\begin{array}{r}\text { Charging } \\
\text { Equipment } \\
\text { types }\end{array}$} & $\begin{array}{l}\text { Standard } \\
\text { Chargers }\end{array}$ & \multicolumn{4}{|c|}{ Rapid Chargers } \\
\hline & $3 / 7 \mathrm{~kW} \mathrm{AC}$ & $22 \mathrm{~kW} \mathrm{AC}$ & $\begin{array}{l}\text { 43kW AC+ } \\
44 \mathrm{~kW} \mathrm{DC} \\
\text { Chademo } \\
+ \text { Combo }\end{array}$ & $\begin{array}{l}43 \mathrm{~kW} \mathrm{AC}+ \\
50 \mathrm{~kW} \mathrm{DC} \\
\text { CHADemo }\end{array}$ & $\begin{array}{l}50 \mathrm{~kW} \\
\text { CHADemo }\end{array}$ \\
\hline Location types & & & & & \\
\hline Public & & 832 & 18 & 14 & 38 \\
\hline Workplace & \multirow{2}{*}{920} & & & & \\
\hline Home chargers & & & & & \\
\hline
\end{tabular}

This situation of mixed connector provision has resulted from the different rapid charging protocols introduced by the EVs coming to market since 2010, when ecars began its infrastructure roll-out. The addition of new funders during the deployment programme enabled the ecars programme to respond to the new technology requirements by installing additional chargers with the new connector specifications.

Other early infrastructure projects, such as NE PIP, have not been able to respond to these new connector requirements due to timing and funding limitations. Therefore, the NE PIP estate only contains one type of DC rapid charging connector, for the Chademo protocol, which was the only specification available in 2010 when the infrastructure was procured.

There have been multiple rapid charge point deployments in larger populated areas within the Republic of Ireland, especially those with large car parks and shopping centres, with capacity for future deployment built into the transmission network system. As the $\mathrm{DNO}^{1}$ for Republic of Ireland, ESB has been able to provide power upgrades where necessary, although this adds significant cost to charge point installation.

The ecars rapid chargers have delivered almost 20 MWh of energy in the Republic of Ireland in the period February 2013 to June 2014. This demonstrates an upward curve of consumption which corresponds to the increase in EV sales, as shown in Figure 3.

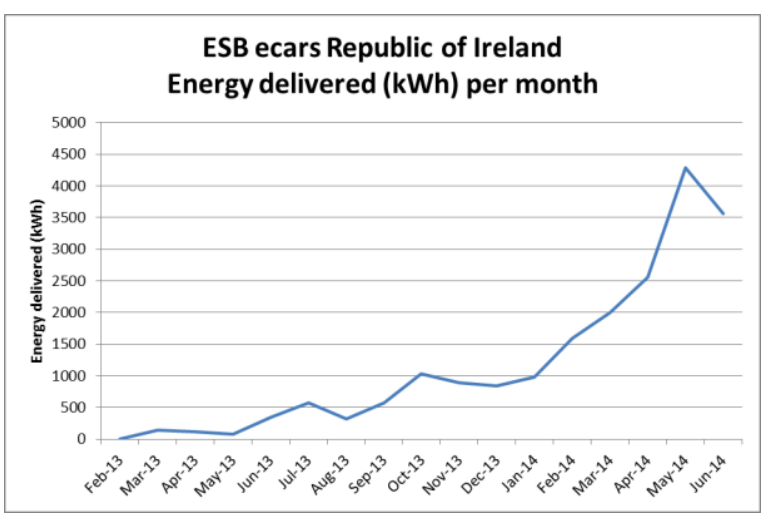

Figure 3: Energy delivered by Republic of Ireland charge point estate to June 2014

Queues of EVs have been reported at certain rapid charger locations in the Republic of Ireland. For example, a second rapid charger has been installed in Dun Laoghaire Dublin in response to excess demand. This location is in the area of highest EV ownership in the country, and the site is on the N11 a key highway between Dublin and Wicklow, which has the third highest EV ownership.

\subsection{RCN}

The 29 RCN multi-standard rapid chargers installed by December 2014 have delivered a total of 5,758 transactions over 7 months. $40,583 \mathrm{kWh}$ of energy was delivered in that period, equating to over 238,000 EV km driven. On average, EV drivers have taken $7.05 \mathrm{kWh}$ of energy per rapid charging transaction, which is comparable with the experience of the NE PIP project in its early years. Recharging transactions are spread evenly through the days of the week, with only a slight increase in demand apparent on Friday.

\footnotetext{
${ }^{1}$ Distribution Network Operators (DNOs) own and operate the distribution network of towers and cables that bring electricity from the national transmission network to homes and businesses.
} 
The relative proportions of use of each of the 3 connector types available on the multi-standard rapid charger is shown in Figure 4. This is broadly in line with the relevant ULEV sales proportions in the UK. However, the use of the Combo protocol is higher than the current ULEV sales proportion would suggest, probably because Combo chargers are much scarcer than Chademo chargers in the UK currently.

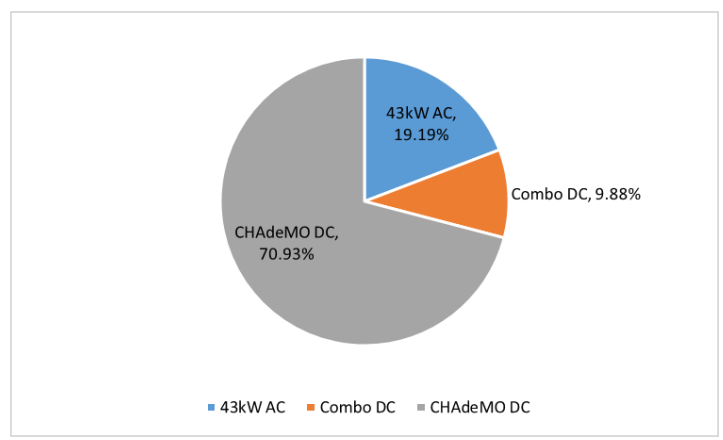

Figure 4 - Proportion of RCN Transactions by charging protocol and connector type.

Usage of this rapid charging estate will continue to be monitored through 2015, as additional chargers are installed. Since over $50 \%$ of the $\mathrm{RCN}$ rapid chargers are to be located along main UK motorways, where both traffic and population densities are high, we shall compare charging behaviour at these sites to behaviour in the less densely populated areas of the defined TEN-T route. This will inform the characteristics of a future business case for public provision of rapid charging facilities.

In parallel with the study of charging data, the RCN project is studying EV trip based data to explore changes in driving and charging behaviour resulting from the availability of rapid charging facilities along key highways. Qualitative studies will also be carried out to gather EV drivers' feedback on factors affecting their behaviour.

\section{Future Business Model requirements}

\subsection{The uncertainty of ULEV predictions}

Any business case for infrastructure must be based on data and reliable forecasts of demand.
Since the demand for recharging services is derived from ULEV sales, the recharging business case depends heavily on predictions for ULEV sales. However, actual ULEV sales in both the UK and Republic of Ireland have been much lower than the forecasts made in 2010. Therefore demand for recharging transactions has been lower than the predictions made, affecting the early business models envisaged for on-going operation and increasing provision.

ULEV sales forecasts are driven by political, commercial and social factors. Politicians may desire a certain proportion of new vehicle sales to be converted into ULEV sales to meet emission reduction targets. ULEV manufacturers have commercial targets to achieve alongside fleet-wide emission reduction targets which have financial penalties. Whilst the customers of vehicles have their own preferences and priorities which may be completely different from the global goals of emission reduction.

In reality it is the production capacity of ULEV manufacturers' which will limit ULEV sales. Whilst it is possible to increase production capacity it is not possible to do this quickly due to both raw material constraints and supply leadtimes, particularly in relation to battery production.

Historical ULEV sales projections were probably informed by ULEV manufacturers' capacity statements, and not by realistic production figures. For example, Nissan's Sunderland plant which manufactures the LEAF and Lithium Ion batteries for all of its European electric vehicle sales, reported production of 17,000[15] EV's during 2014. This is clearly a long way from its stated annual production capacity of 50,000 LEAF[16]. Since the LEAF is reported to be Europe's best selling EV, future sales forecasts should be modified to reflect more realistic volumes for effective use in the recharging business case.

Currently there are four main European EV manufacturers in the marketplace; BMW, Renault, Nissan and VW. Non-European manufacturers Mitsubishi and Tesla also contribute to volume European sales, with other manufacturers making smaller contributions. A review of grey literature suggests that the four main European manufacturers have a combined annual EV production capacity of approximately 220,000. Based on this estimate of production capacity, if European EV sales increase at a rate of $30 \%$ per 
year, production capacity will become a constraint from 2020. But if sales grow at 50\% per year, this position will be reached by 2017 .

Over stating ULEV sales forecasts leads to problems for both the ULEV marketplace and for recharging service providers. Firstly, press reports comparing actual sales figures against unrealistic forecasts lead the public to believe that the market is unsuccessful. The already difficult challenge of persuading the public of the genuine merits of ULEV transportation are adversely weighted by such reports. Secondly, recharging network operators and infrastructure owners are in danger of building infrastructure based on unrealistic demand expectations.

This unrealistic expectation of demand is further compounded by the expectations set by the EU's proposal on the deployment of alternative fuels infrastructure[2]. This proposal calls for binding quantitative targets to be set by each European member state governing the roll-out of recharging facilities. The problem is that demand for such services is linked both to actual ULEV sales, and to the characteristics of the recharging facilities available, and should therefore be driven by the market in order to make it sustainable, not by unrealistic ULEV sales targets. The EU's proposed target would require the UK to provide 1.21 million recharging points by 2020, almost 200 times more than the installed base in 2014. This figure is based on national targets for ULEV sales, which are currently inaccurate. Furthermore, it requires that $10 \%$ of these must be publically accessible. As the results described in this paper show, user behaviour varies depending on charge point characteristics such as location, capacity, access, and fees. Therefore, user recharging behaviour should be used to predict public charge point requirements, not just ULEV forecasts.

The EU's suggestions for funding this level of provision through the use of building, parking and environmental codes for businesses are currently unrealistic. The current demand for public recharging transactions from EV drivers is not high enough to fully cover the economic costs of capital, installation, operation and maintenance. The actual uptake of ULEVs and therefore demand for recharging transactions has been lower than the predictions made in 2010, affecting the early business models envisaged for on-going operation and increasing provision. The consequence is that the majority of charge point owners studied for this paper are continuing to financially support the costs of operation of recharging infrastructure and cannot yet foresee an acceptable conventional business model.

Therefore we can conclude that there is currently no feasible, purely economic business model for the provision of public recharging infrastructure in the UK or Republic of Ireland.

\subsection{The charge point owner's perspective}

The charge point owner is now at the centre of a system of infrastructure which is seen as essential by many stakeholders such as government, environmental bodies and EV drivers, and they are expected to at least maintain the current level of supply. However, the expectations and business assumptions upon which most hosts made the decision to adopt charge points, have not materialised.

Charge point owners in NE England are a diverse group of over 60 organisations who have decided to provide recharging infrastructure for a variety of political, economic, social and environmental reasons. Peer pressure is also a relevant factor in the region because of the high profile role the $\mathrm{NE}$ has played in low carbon vehicle development to date. The region is also home to the Nissan European LEAF production and battery plants.

The Climate Change Act[17] is driving the implementation of sustainable transport solutions in the UK in order to reduce domestic greenhouse gas emissions. UK LAs are therefore being encouraged by political motivations to provide appropriate services, such as EV recharging infrastructure. This represents a model of provision for social good, as opposed to the likely economic motivations of a private business. LAs may have three target audiences in which the provision of recharging infrastructure should encourage low carbon vehicle uptake; the general public, its own employees, and its own fleet. Private Businesses on the other hand would be targeting only their employees and own fleets. There is also a third category of NE host such as Universities and Health providers which have some social and environmental targets leading them to offer recharging services to visitors as well as employees, but not to the general public. 
Since ESB ecars and the RCN chargers are all owned by a single organisation with national coverage and a combination of commercial and social and environmental objectives, their perspective may be slightly different from the NE region. This will be studied further.

However, the customers wishing to use the recharging services, EV drivers, may have very different demands to those assumed by the charge point hosts. It is therefore important to consider the views of this key stakeholder group separately.

\subsection{The EV driver's perspective}

EV drivers' demands for public recharging infrastructure are based on cost, ease of use reliability and location. The additional services provided such as free parking have been a key determinant in NE charge point city centre usage to date. The available capacity and reliability of charge points will also become increasingly important as EV volumes increase and in certain locations with dense EV populations this has already become a problem, for example in Republic of Ireland and on the UK's Electric Highway. Increased provision of rapid charge points has been seen to resolve this problem in some cases, but this brings associated increases in set-up and operating costs as it encourages greater use. The need for additional charge points also brings the challenge of limited power capacity and the high costs associated with increasing supplies is not to be underestimated by hosts.

\subsection{The role of subsidies}

All of the early-to-market EV recharging estates described in this paper were created and operated under public subsidy, in order to seed the marketplace for further EV and recharging equipment adoption. In NE England, the availability of NE PIP grant funding towards purchase and installation costs heavily influenced hosts decisions to adopt charge points. These grants have now ended so one hypothesis is that provision will not increase further without new government subsidies being introduced. Indeed, since 2013 only a small quantity of additional, mainly rapid, charge points have been added to the regional network, the majority of which were funded by national OLEV and local authority subsidies.
The NE PIP project also paid all system operating costs until June 2013, so charge point owners were therefore shielded from the true costs of operation. The result is that NE charge point owners have adopted recharging infrastructure without being fully aware of the costs of operation. As a consequence, some charge points have been switched off, some have not been repaired and others have been moved to new locations where demand is higher.

As public subsidies decline, the infrastructure owners must find other ways to cover the on-going costs of operation and to recover the capital investments made, in order to provide a continuing service to EV drivers.

\subsection{The role of fees for $\mathrm{EV}$ recharging}

As charge point owners are increasingly exposed to real operating costs they will also become aware of the new commercial opportunities available to them, such as fees, marketing and the provision of associated services. The introduction of fees for EV recharging is likely to affect the behaviour of EV drivers in terms of their recharging habits (time, location, duration etc.), willingness to pay, journey characteristics and potentially their overall EV usage. These behavioural changes will, in turn, affect the owners of recharging equipment and the businesses operating this equipment in recharging networks (Network Operators).

Increased understanding of the operating costs is likely to drive assessments concerning fees and further recharging provision in different directions to those taken against the backdrop of public subsidy. Type, quantity and location of recharging equipment are all key determinants in this business model. Therefore studies of comparative usage data from different types of recharging equipment and charge point location types should be used to inform future fee structures.

\subsection{The way forward - a broader measurement model ?}

Charge point owners are now faced with five elements influencing the business model for operating their recharging infrastructure; Charge point features, ULEV features, EV drivers' requirements, charge point technology and recharging technology. Each of these areas contains unknown and inter-related factors and therefore requires predictions based on market 
intelligence to date and informed extrapolation. Many charge point owners do not have the capacity or business need to carry out this in depth work, and are therefore at risk of making uniformed decisions.

NE charge point owners have varying reasons for providing recharging infrastructure, including political, social and environmental objectives many of which cannot be measured in purely economic terms.

There is therefore the potential for some form of social and environmental accounting model to be developed which will provide an alternative accounting framework for the provision of public recharging infrastructure. A social account that could include the use of social return on investment would enable a charge point owner to assess and report its impact on society and the environment, alongside its economic measures. A flexible framework could be used to collect, analyse and interpret quantitative and qualitative data resulting from the provision of recharging infrastructure. Use of such a framework would enable charge point owners to understand and evaluate the wider value provided by recharging infrastructure and its services, and to value how it affects the people, environment and resources they are responsible for.

Further research is ongoing to build and assess such a framework. An analysis of charge point owners' attitudes towards their recharging infrastructure will be performed, in parallel with the authors' continuing longitudinal study of the usage of NE charge points. This will enable us to develop a social and environmental account from the perspective of a charge point owner and to reflect within it the voice of the EV user as a key stakeholder.

\section{Conclusions}

Subsidies have supported the uptake of ULEV in both UK and Republic of Ireland to date. However the ULEV manufacturers will always determine production capacity and country allocation quantities. Therefore, the size of the ULEV population is determined by the attractiveness of the package of subsidies available, but is also constrained by the production capacity which the ULEV manufacturers create for each model. More reliable ULEV forecasts are required to inform the business model for recharging infrastructure.

The study to date shows clear recharging habits prior to subsidies being withdrawn, however we are now experiencing unintended consequences of subsidy policy on driver behaviour and consequently difficulties in the development of future sustainable business models. Both charge point owners and EV drivers are key stakeholders in the development of this business model, but they have widely differing viewpoints.

User recharging behaviour varies depending on charge point characteristics such as equipment type, location, capacity, access, and fees. Therefore, data produced from actual recharging behaviour should be used to predict future public charge point requirements, as well as reliable ULEV sales forecasts.

A social and environmental accounting framework may provide a suitable business model for some infrastructure owners, however others may require to make purely economic business decisions. Following the success of UK policy to encourage EV ownership to date, there is now a real risk that customers may perceive that the recharging infrastructure is not developing sufficiently to support the wider ULEV adoption required. This presents a major risk that the gains made to date will be lost if new business models are not developed to support the operation of existing infrastructure and to fund future new infrastructure.

A flexible framework may enable charge point owners to demonstrate and account for the social, environmental and economic impacts they have made by providing recharging infrastructure, thereby opening up alternative business models for ongoing and increasing future provision, which in turn would benefit EV users.

\section{References}

[1] European Commission. (2013). Clean Power for Transport. Available: http://ec.europa.eu/transport/themes/urban/cpt/ind ex_en.htm

[2] European Commission, "Proposal for a DIRECTIVE OF THE EUROPEAN PARLIAMENT AND OF THE COUNCIL on the deployment of alternative fuels infrastructure ", ed. Brussels, 24.01.2013. 
Office for Low Emission Vehicles, "Plug-in Car Grant," ed. https://www.gov.uk/government/publications/p lug-in-car-grant, 2011.

[4] Office for Low Emission Vehicles, "Plug-in Van Grant," ed. https://www.gov.uk/government/publications/p lug-in-van-grant, 2012.

[5] Office for Low Emission Vehicles. (2010). Plugged in Places. Available: https://www.gov.uk/government/publications/p lugged-in-places

[6] Office for Low Emission Vehicles, "Driving the Future Today - A strategy for ultra low emission vehicles in the UK," OLEV, Ed., ed. London, 2013.

[7] $(19 / 02 / 2015) . \quad E S B . \quad$ Available: http://www.esb.ie/main/home/index.jsp

[8] (13/02/2015). ESB ecars. Available: http://www.esb.ie/electric-cars/index.jsp

[9] (20/05/2014). The Rapid Charge Network project. Available: http://rapidchargenetwork.com/

[10] Y. B. Hübner, P; Hill,G; Neaimeh,M;, Austin,J; Gray,L; Herron,C; Wardle, J; , "49,999 Electric car journeys and counting," presented at the EVS27, Barcelona, 2013.

[11] Charge Your Car Limited. Available: http://chargeyourcar.org.uk

[12] Setting emission performance standards for new passenger cars as part of the Community's integrated approach to reduce $\mathrm{CO} 2$ emissions from light-duty vehicles, THE EUROPEAN PARLIAMENT REGULATION (EC) No 443/2009, 23 April 2009.

[13] A. E. A. M. Association. (2015, 22/05/2015). Electric vehicle registrations - 2014 (05/02/2015). Available: http://www.acea.be/pressreleases/article/electric-vehicle-registrations$\underline{2014}$

[14] Department for Transport. Vehicles statistics [Online]. Available: https://www.gov.uk/government/collections/ve hicles-statistics

[15] C. Ford. 22/02/2015). Demand for Nissan LEAF and Qashqai sees more than 500,000 cars produced in 2014. Available: http://www.thejournal.co.uk/business/businessnews/demand-nissan-leaf-qashqai-sees$\underline{8397402}$

[16] (2010, 22/02/2015). NISSAN TO BUILD LEAF ELECTRIC VEHICLE IN SUNDERLAND (18th March 2010). Available: http://www.nissan.co.uk/GB/en/insidenissan/news/leaf_news/ev_news.html

[17] Department of Energy and Climate Change, "Climate Change Act 2008," DECC, Ed., ed. http://www.legislation.gov.uk/ukpga/2008/27/p dfs/ukpga 20080027 en.pdf.

\section{Acknowledgments}

The authors gratefully acknowledge the support of; OLEV's North East Plugged in Places project, ESB and the UK's Engineering \& Physical Sciences Research Council and the Economic \& Social Research Council funding of iBUILD (Infrastructure BUsiness models, valuation and Innovation for Local Delivery) - EP/K012398/1 (http://research.ncl.ac.uk/ibuild/). The authors also acknowledge the ongoing support of the EU's TEN-T programme, UK DfT, and the RCN project partners along with Ecotricity the operator of the Electric Highway.

\section{Authors}

Josey Wardle is Infrastructure Manager at Zero Carbon Futures, and is undertaking a $\mathrm{PhD}$ looking into the future for EV charging. A Manufacturing Engineer with 20 years' experience, Josey managed NE England's Plugged in Places project and the UK's Rapid Charge Network project delivering and monitoring over 1,000 EV charge points to date.

Professor Phil Blythe is Professor of Intelligent Transport Systems and Director of the Transport Operations Research Group. Phil is internationally recognised as an early pioneer of ITS research and is currently Chair of the IET's Electric Vehicle Steering Group.

Dr Jane Gibbon is a senior lecturer in accounting and researches the practice of social accounting using action research approaches. Jane is both an academic researcher and practitioner working with third sector organisations on their social accounts. Jane is an ESRC Third Sector Research Fellow, a member of the Centre for Social and Environmental Accounting Research (CSEAR) and a member of the Social Audit Network (SAN).

Dr Colin Herron is MD of Zero Carbon Futures, which delivered the EV charging infrastructure for the North East of England. Colin has over 40 years' experience in the automotive industry, with a current focus on Ultra Low Carbon Vehicle development and associated technology.

Dr Yvonne Hübner is the Inward Investment Manager for Science Central, where she delivers projects on digitally enabled urban sustainability. Previously, Yvonne worked as a Senior Research Associate on the SWITCH-EV electric vehicle trial in the Northeast of England and was part of the management teams of realworld demonstration trials such as smartCEM, Compass4D and RCN. 